\title{
PART II. CURRENT CONCERNS IN THERAPEUTIC RESEARCH
}

II.1. Persistent Myco. leprae

II.2. Drug Resistance

II.3. Combined Therapy

II.4. Transfer Factor

\section{II.1. Persistent Myco. leprae}

Prof. Saerens

I should like to ask Dr Rees about the problem of persistent bacilli. You presented three series of patients treated for 6 months, 12 months and 18 months, and I was more or less struck by the impression that the longer therapy was continued the greater the percentage of persisters, which seems paradoxical on the face of it. Compared with the situation in tuberculosis we can say that the longer the therapy, the higher the risk of resistant mutants appearing. You showed that in three cases the bacilli were still sensitive. We shall have to wait for further data, but may we ask whether these three cases belonged to the same group and whether you have any further comment on these data?

\section{Dr Rees}

You are correct, Dr Saerens, to date our data show a higher proportion of "takes" in mice inoculated with bacilli from patients after receiving two years of rifampicin, compared with bacilli recovered from patients receiving rifampicin for 12 or 6 months. However, the numbers are small and are not statistically significant. Moreover, bacilli isolated in mice from three patients are all sensitive still to rifampicin, albeit these strains have come from patients treated for only six months with rifampicin. While I would agree that it would be more likely for resistance to be manifested in isolates from patients treated up to two years, the results of these tests are not yet available. While at this early stage of our studies we cannot rule out the possibility that some bacilli we are isolating are rifampicinresistant, I must remind you that in this special study all the patients are receiving rifampicin plus thiambutosine. By giving combined therapy it is unlikely that drug resistance to rifampicin will occur.

\section{Dr Ellard}

I would like to make a few comments concerning the continued persistence of viable drug-sensitive leprosy bacilli in sites such as peripheral nerve and striated muscle despite long-term treatment with dapsone or rifampicin. All the evidence suggests that both drugs readily diffuse into most body tissues, and in a recent experimental study both drugs were shown to penetrate readily into the sciatic nerves of the dog and sheep. It must therefore be concluded that the persistence of these viable drug-sensitive leprosy bacilli cannot be due to inadequate tissue penetration of either drug. A more probable explanation is that a significant proportion of the leprosy bacilli in such tissue sites are dormant and as a consequence are physiologically resistant to killing by either drug. Dapsone is in any case primarily a bacteriostatic drug, while it is known that rifampicin has very little bactericidal activity in vitro against non-growing Myco. tuberculosis. This aspect of the chemotherapy of leprosy may therefore be similar to that encountered in the chemotherapy of tuberculosis, where it is apparent that drugs 
such as isoniazid and rifampicin that are highly bactericidal against actively growing Myco. tuberculosis are unable to kill dormant bacilli. Experience in the treatment of tuberculosis however indicates that the drug pyrazinamide is capable of killing near-dormant tubercle bacilli. Unfortunately experimental evidence indicates that when doses of pyrazinamide are given that are well tolerated in man, the concentrations of the drug attained in the body fail to prevent multiplication of Myco. leprae in the mouse footpad. I would suggest therefore that one of the most important areas of chemotherapy research would be to try and find an analogue of pyrazinamide with significant activity against Myco. leprae.

\section{Prof. Freerksen}

The opinion that antibacterial substances might have no influence on "dormant bacilli", is widespread but never proved. Physiological saline is no culture medium, in which bacteria could multiply. They thus remain "dormant" as shown in Table 1.

TABLE 1

Subcultures after action of rifampicin + isoniazid in different doses after 14 days' contact

\begin{tabular}{|c|c|c|c|c|c|}
\hline \multirow{2}{*}{$\begin{array}{c}1 \mathrm{mg} / \mathrm{ml} \\
\text { in } \\
\text { Myco.tub. } \mathrm{H}_{37} \mathrm{Rv}\end{array}$} & \multirow{2}{*}{$\begin{array}{c}/ \mathrm{ml} \\
\mathrm{INH}+\mathrm{RAMP}\end{array}$} & \multirow{2}{*}{$\begin{array}{l}\text { time of } \\
\text { contact }\end{array}$} & \multicolumn{3}{|c|}{$\begin{array}{c}\text { Subcultures }\left(0.1 \text { of } 10^{0}\right) \\
\text { on Löwenstein-Jensen egg-medium* }\end{array}$} \\
\hline & & & after 3 & 4 & 6 weeks \\
\hline Saline & $\begin{array}{r}100+100 \\
50+50 \\
10+10 \\
5+5 \\
0+0\end{array}$ & 14 days & $\begin{array}{l}----- \\
----- \\
----- \\
----- \\
+H+H+H\end{array}$ & $\begin{array}{l}----- \\
----- \\
----- \\
----- \\
\mathrm{H+W}+\mathrm{WH}+\mathrm{H+}\end{array}$ & $\begin{array}{l}----- \\
----- \\
----- \\
----\bar{H} \\
\mathrm{HH} H \mathrm{HW}\end{array}$ \\
\hline $\begin{array}{l}\text { Lockemann } \\
\text { medium }\end{array}$ & $\begin{array}{r}100+100 \\
50+50 \\
10+10 \\
5+5 \\
0+0\end{array}$ & 14 days & $\begin{array}{l}----- \\
----- \\
--\cdots-\cdots \\
--\cdots-\cdots \\
\mathrm{H}+\mathrm{H}+\mathrm{H}+\mathrm{H}\end{array}$ & $\begin{array}{l}----- \\
----- \\
----- \\
----- \\
+4+4+4+4+4+4+4\end{array}$ & $\begin{array}{l}----- \\
----- \\
----- \\
----- \\
+H+H\end{array}$ \\
\hline
\end{tabular}

This very simple but clear-cut experiment shows that isoniazid + rifampicin will have a bactericidal effect in bactericidal concentrations, even if the bacteria have no multiplication metabolism, that is they are "dormant". No matter if the first culture has been given into a medium or physiological saline. The "dormant bacilli" without multiplication-metabolism were killed in this experiment, too.

Whether antileprosy drugs exert any action upon "dormant bacilli", cannot be ascertained in vivo, and whether a substance has a bacteriostatic or bactericidal effect, not in animal tests. The reaction between bacterium and macro-organism leads finally from bacteriostasis to bactericidal activity. This process is supported and accelerated by chemotherapy; otherwise we all would be no longer above ground; virulent micro-organisms would have eaten up all macro-organisms!

\section{Dr Ellard}

All I can say is that the ability of single drugs to kill Myco. tuberculosis in vitro has been demonstrated conclusively by numerous groups of workers. Furthermore since several groups have shown that rifampicin specifically inhibits bacterial 
DNA-dependent RNA polymerase, it is clear that its antibacterial activity is dependent on the bacteria being in a state of active growth.

\section{Dr Browne}

From the point of view of the public health worker and the clinician, persistent bacilli are not a problem, but they are a problem to the individual patient who may relapse and thereafter become a public health problem. We are convinced that the active drugs, despite the proliferation of a certain number of persisters, are active in leprosy and will help the individual patient.

\section{2. Drug Resistance}

\section{Dr Browne}

Another problem is posed by the increasing occurrence of drug resistance in the world. Sooner or later whether we work in Malaysia or in Britain we shall have to face this problem. It is becoming increasingly serious, as we were reminded yesterday. Fortunately we have two drugs that up to the present have been able to control bacillary proliferation in those patients showing dapsone-resistant bacilli. But the day will come when we shall have resistant forms due to clofazimine and rifampicin.

\section{Dr Pearson}

There is quite a lot of drug resistance; I am thinking particularly of dapsone resistance. We have a series of about 100 proved cases in Malaysia; about 140, mostly not proved, but clinically of the same pattern, in the clinic where I work in Addis Ababa. The interesting thing is the time that it takes, 10 years, 15 years, 20 years from the start of treatment for relapse to appear, for resistant strains to multiply and emerge. There is some suggestion that in Ethiopia lower dosage of dapsone has been used in general in leprosy treatment than in Malaysia and that the clinical signs of drug resistance come out sooner. The mean time is about 7 or 8 years in Addis Ababa, about 15 years in Malaysia. In out-patient control centres irregular treatment is also of course more likely, and therefore dapsone resistance is more likely to happen in patients under out-patient therapy. The important thing it seems to me is first of all to get an estimate of how serious the problem is. Our current figures suggest that somewhere round about $5 \%$ of patients with lepromatous leprosy, sooner or later will probably get dapsone resistance. This is, of course, very provisional, but such numbers are sufficient to make a major impact on the management of lepromatous leprosy in a big leprosy treatment centre. Drug resistance only seems to happen in lepromatous cases, presumably because it is only in lepromatous leprosy that there is a sufficiently high bacillary population for there to be a reasonable number of spontaneous mutants initially.

\section{Dr Browne}

I think your figures of $5 \%$ will certainly have to be raised. The percentage of those patients who were in the mid-forties' drug trials in Carville, Louisiana, and now have drug resistant forms is about $50 \%$. I think a patient of mine still holds the world record-four years and four months from the initiation of treatment to the development of proven resistance. 


\section{Dr Pearson}

One thing to add is that the important thing is prevention, and I am convinced that lepromatous patients should be started off at least on dual drug therapy in an attempt to reduce the incidence of dapsone resistance.

\section{Dr Browne}

In how many countries in the world is it possible to afford such therapy? Leprosy is only one of the many problems confronting these poor developing countries.

\section{Prof. Pattyn}

I think that we should refer to what we have learned from tuberculosis. I invite all those who are not "contaminated by tuberculosis", as you said yesterday, to get contaminated as soon as possible and to read something about how the modern treatment of tuberculosis was found out, why it was established and why it should be as it is. As has just been said in multibacillary cases it is a "must" to start treatment with combined therapy in order to prevent resistance. The only difference is that leprosy has such a long generation time, and instead of taking one year, more or less, to become evident as in tuberculosis, resistance in leprosy takes a decade.

\section{Dr van der Meulen}

I would like to ask Dr Karat if he thinks that early detection of resistance will be possible by examining slides of bone marrow, because he said yesterday that bacilli will remain viable much longer in the bone marrow.

\section{Dr Karat}

One can certainly demonstrate the bacilli in the bone marrow, and as I tried to indicate earlier, we could not find a clear relation between the staining characteristics of those bacilli and their viability. If one is looking for "persisters", certainly bone marrow will be one site which one should seriously consider.

\section{Dr Urbancik}

In tuberculosis we have already got in Germany in some laboratories about 6 to $8 \%$ of rifampicin resistant strains. If rifampicin is going to be administered in leprosy on its own, we can probably expect resistant strains of Myco. leprae within a few years.

\section{Dr Rees}

I entirely agree with the previous speaker that from the vast experience in the field of tuberculosis which has shown that monotherapy inevitably results in a high incidence of drug resistance, initial monotherapy in the treatment of lepromatous leprosy by rifampicin, or for that matter any new antileprosy drug, is unjustifiable as routine treatment for an appreciable number of patients. I would like to reinforce this recommendation by briefly recapitulating the present picture of dapsone resistance in leprosy which is only beginning to unfold. From the data I and Dr Pearson have presented from detailed studies on drug resistance in Malaysia and Ethiopia respectively, including proof from dapsone sensitivity tests using the mouse infection model, it is clear that: (1) While relapse due to the emergence of dapsone resistance in a minority of patients presents as early as 3-5 years, in the vast majority the mean time to emergence of drug resistance is 
many years-probably 15. In Malaysia where dapsone has been used systematically for 25 years, even in the patients maintained for this number of years on dapsone, some are still relapsing with dapsone resistant leprosy. (2) There is increasingly good scientific evidence that the incidence of sulphone resistance is higher in irregularly treated patients. (3) There is equally good evidence that the incidence of sulphone resistance is higher in patients receiving lower doses of dapsone (lower than $100 \mathrm{mg}$ daily) or where lower doses have been administered by treatment with some of the di-substituted sulphones, such as sulphetrone, where all such derivatives are equivalent to giving 5-20 mg of dapsone. The picture presently presented of dapsone resistance is based entirely on monotherapy, which initially seemed perfectly justifiable because early relapses did not occur. The more recent revelation of the very long incubation period preceding the occurrence of dapsone resistance is as pernicious and frightening as was the revelation and realization of the prolonged exposure necessary for revealing the carcinogenesis of many environmental factors, industrial chemicals or drugs. There can be no doubt that we are only beginning to inherit an ever-increasing dapsone resistant problem, and although resistance to thiambutosine and thiacetazone becomes apparent within 2-3 years, it cannot and must not be assumed that the prolonged period of evolution will be unique to dapsone. It could apply to rifampicin or to any other new antileprosy drug introduced. It should be the duty of all of us responsible for future developments in the chemotherapy of leprosy to insist on initial combined therapy for all new patients with lepromatous leprosy.

\section{Dr Browne}

Would anybody like to comment on the recommendation that patients with lepromatous leprosy should continue treatment for life, after apparent clinical and bacteriological quiescence has been achieved? Would you expect there to be a greater proportion of resistant cases as result of this therapy?

\section{Prof. Freerksen}

"Resistance" has no absolute rate, but means a gradually differentiated sensitivity restriction. The problem therefore is whether the existing sensitivity is high enough if compared with the applied dose of an antibacterial substance. When stating that a patient is resistant against dapsone one must therefore mention at the same time the doses of the antibacterial substance administered. There may for instance be resistance against dapsone at a dosage rate of $0.1 \mathrm{mg} / \mathrm{kg}$ body weight, but sensitivity at $1 \mathrm{mg} / \mathrm{kg}$. Resistance occurs more easily when bacteriostatic agents are used in small doses. It is therefore a great mistake to administer too low doses of antibacterial substances. Big problems also arise with regard to the statement that the effectiveness of a therapy determines its duration. The more intensive the therapy and the smaller the number of germs, the shorter the treatment time. Patients pretreated with dapsone for years or even decades who were still bacteriologically positive at the outset of the therapy we recommended are a distinct proof that the therapeutic effect of dapsone was unsatisfactory.

Our therapeutic results show that bacteriologically negative results can be obtained at variable intervals. There were patients who already became negative after two months and remained so during the observation period. And there are others who are still positive after a treatment period of two years. We do not know the reason for this phenomenon. It may be possible that the patients have not swallowed the medicament given to them, but this seems not to be the only 
reason. I personally think that it is useless to apply the same therapy over a period longer than two to three years. If a patient is still bacteriologically positive at the end of those two or three years of treatment, the therapy should be changed.

\section{Dr Browne}

Should we continue with an effective treatment, after clinical and bacteriological quiescence has apparently been achieved?

\section{Prof. Freerksen}

According to our experience the time necessary for treatment differs from one patient to another. It therefore has to be decided in each individual case at what time treatment should be stopped. This is very difficult, since we do not possess any absolutely relevant criteria characterizing a successful cure in each individual case. At the present level of our experience, we must have the courage to terminate a treatment after a sufficiently long observation period during which the patient remains negative and to investigate then thoroughly the occurrence of relapse. This, of course, must be done in hospitals chosen for this purpose and with the help of suitable doctors. This method is justified as long as a patient can be observed for a period of two to five years. In the case of relapse the patient must of course be treated again. After a short-term treatment this does not offer any problem, since the bacteria remained sensitive.

\section{Dr Rees}

Dr Browne has posed a logical and practical question. It is this-bearing in mind that among patients treated with dapsone for many years there is evidence that some will relapse with dapsone resistance, others apparently harbour a few persister, but viable organisms, that when treatment is stopped will eventually result in the recurrence of active disease. In the latter case the infection is sulphone sensitive and the patients will again respond to dapsone therapy. His question is basically whether these two possible deleterious outcomes will more likely be overcome, or enhanced, by prolonging dapsone therapy indefinitely. Before attempting to answer this question I must stress that these problems only apply to patients with lepromatous leprosy and that from the studies of our own group in Malaysia we entirely agree that both possibilities do occur. However, while both phenomena can occur in lepromatous patients treated with dapsone, fortunately they only occur in a proportion, and since there is no routine investigation for predetermining such cases we can only live with the problem and not prevent it in patients that currently are at this stage of therapy. Therefore, the short answer is to continue maintenance doses of dapsone indefinitely in lepromatous patients already started on sulphone therapy. However, for all newly identified patients with lepromatous leprosy we should be able to prevent the emergence of dapsone resistance by initiating them on a course of dapsone combined with another antileprosy drug and then followed by dapsone alone. Such combined therapy will, on the other hand, not necessarily obliterate a residual persister population of viable organisms, which are drug sensitive, and will multiply when therapy is stopped. The question of whether such drug sensitive persisters can ever be completely eradicated in all patients with lepromatous leprosy still remains a question of the future. Hitherto, dapsone and other antileprosy drugs are believed to be predominantly bacteriostatic and therefore a bactericidal drug, such as rifampicin, may obliterate such persisters, and only 
further prolonged studies will answer this important question. If rifampicin or other bactericidal antileprosy drugs fail to do so, then it is likely that a proportion of patients with lepromatous leprosy will never be sterilized by chemotherapy alone and will require, if such a procedure can be devised, a form of immunotherapy which in combination with chemotherapy will enable the host to contribute to the eradication of persisters.

\section{Dr Walter}

Life-long treatment has partly been recommended by WHO because we have no alternative. At the time being we really have only one simple first line drug available. All other drugs for practical purposes cannot be used at the moment for a period of three, four or five years for reasons which we don't have to spell out in detail here. We know definitely that the majority of patients who have been treated regularly for a period of five years at an average, become negative by routine methods. How far they are negative to the last bone, the last muscle we don't know, but we assume they are not. So we have to go on treating them.

\section{II.3. Combined Therapy}

\section{Dr Browne}

I should like us to spend a short time debating the pros and cons of combined therapy. Does combined therapy postpone the appearance of resistance, does it reduce the duration of infectivity, or the duration of treatment; does it make for rapid bacillary clearance and does it prevent or indefinitely postpone the onset of peripheral nerve damage? These are questions that have long troubled those working in the clinical field, and it has been suggested that combined therapy will help. What could we recommend for further investigations?

\section{Dr Molesworth}

I should like to say just a word about some earlier experience of combined therapy. Somewhere about 1955 in Malaya, we tried one series of 25 untreated patients on dapsone, another on thiacetazone (TB1), each drug alone, and finally a third series on the two drugs in combination. We gave marks only for those who improved bacteriologically, clinically and histopathologically. I was working at the time with Dr Hale from Singapore University and he did the biopsies while I did the BI. Now we found that in the dapsone group six cases out of 25 had shown improvement in all three aspects, in the TBl group three, and of the combined treatment group, 14. Whenever I have found a case on dapsone, the BI has fallen steadily but sooner or later has stopped and then continued without further fall; when we have added thiacetazone, or as we do in Malawi at the moment, a combination tablet of isoniazid and thiacetazone, the period of infectivity is shortened, the bacillary load falls and the patient progresses. We are very strong advocates for combined therapy. Particularly do I insist on this in Malawi where we have got over 200 cases on DADDS.

\section{Dr Ramanujam}

In our treatment of lepromatous cases for the past 20 years we have come across an occasional case where in spite of the patient receiving adequate sulphone 
therapy under controlled conditions, there has been some progress, but later on the progress ceased with no further fall in the Bacteriological Index as just mentioned by Dr Molesworth. The only other drug we could ever consider and which we could combine with dapsone was thiacetazone, and we did this in quite a number of cases. I cannot give you the exact figures, such as the maximum dose of dapsone the patients had received previously; but we administered thiacetazone in a dose of 100-150 mg per day in a single dose. All these cases have registered considerable clinical improvement, although bacteriologically the improvement is rather slow. We have not encountered side-effects of thiacetazone as observed especially in the treatment for tuberculosis. Under the existing conditions in India we find it very useful to combine dapsone with one or two other substances in patients who do not respond as we would have expected them to do.

\section{Prof. Freerksen}

We should not simply talk about combined therapy as such, but always specify which combinations of drugs are meant. Not all combinations are good. Since we never have a sufficient number of equal, comparable cases, it cannot be demonstrated by the usual clinical trials that a certain combination is more valuable than another or better than a highly effective single substance. An investigation with 10 or 20 cases divided into several groups, is not worth-while. The simplest trial must be made up of three groups (untreated cases, treated cases and control group) consisting of at least 30 cases each, i.e. about 100 patients altogether. None of us would be in a position to carry out such a trial.

\section{Dr Karat}

I have a few observations to make on combined therapy. First I will take up thiacetazone. As Dr Ramanujam mentioned, this is a drug which is easily available in India and is very cheap. That was the reason why it was chosen for study, secondly it was widely accepted in the domiciliary treatment of tuberculosis. The design of the study was to compare three groups of patients: (1) standard treatment with dapsone, $100 \mathrm{mg}$; (2) dapsone plus thiacetazone; and (3) thiacetazone and isoniazid. Few observations reviewing our findings; first, we could not record any significant difference between the three groups of patients I have now described in relation to elimination of bacilli. Secondly, there was a marked increase in peripheral neuropathy in patients treated with the combination of thiacetazone and isoniazid as compared to the other groups. The figures approached $10 \%$ and the patients under study were of the order of 200 , so that the findings are significant.

I should like to refer to one other combination of drugs which we have used on occasion. This was in highly bacilliferous untreated lepromatous leprosy patients who presented with severe respiratory symptoms and some of whom had ulcerating leprous nodules. In this context three months' study of dapsone versus dapsone and daily $1 \mathrm{~g}$ streptomycin produced a striking difference (a) in the clinical resolution of lesions, (b) in the amelioration of respiratory symptoms, (c) in the fall in Morphological Index and (d) in the fall in the Bacterial Index. The progress, as far as the Bacterial Index was concerned, seemed to attain a plateau between three and six months from the onset of treatment.

\section{DrPearson}

I think one must ask why we are using double therapy. There are two possible reasons. The first is the possibility of getting a quicker cure, a quicker response. 
On analogy with tuberculosis, I understand that this is unlikely. When two drugs are used against tuberculosis the initial response I believe is no more rapid than that of the best one used alone. Two drugs are used in order to prevent the emergence of resistance. This is the second reason for using combined therapy, and again by analogy with tuberculosis, it seems to me highly probable that it will have this effect against $M y c o$. leprae also. I can see no reason why double therapy in leprosy should be less effective than double therapy in tuberculosis. But this applies merely to lepromatous leprosy. There are plenty of data for us to know that in non-lepromatous leprosy, drug resistance is likely at worst to be only a very occasional, rare phenomenon.

\section{DrRees}

May I take a few minutes of this discussion to present a number of general and particular basic principles in bacteriology and chemotherapy which are relevant to our present discussions on drug resistance in leprosy?

The first refers to choosing alternative drugs for relapsing patients. Several papers have been presented at this Colloquium where such patients have been given alternative drugs without considering the basic principles of cross resistance between drugs of similar chemical structure and mode of action. Thus for the Chemotherapy of leprosy, dapsone and all other sulphone derivatives, long-acting sulphonamides and acedapsone (DADDS) can be grouped together as having a common mode of action against Myco. leprae. Therefore, any leprosy patient who relapses under rigorously supervised treatment with one of any of these drugs in this group will not benefit from any other of the drugs in this same group because they have the same mode of action and will show cross resistance. The same basic principle applies to the thioureas i.e. thiambutosine and thiacetazone, with similar essential chemical structures and mode of action, and therefore a patient resistant to one will show cross resistance to the other member of this thiourea series. On the other hand, the mode of action of the thioureas is completely different from that of the sulpha-group of drugs and therefore there is no cross resistance between the two groups, and they are compatible alternative groups of drugs. On present evidence and known modes of action the three other important antileprosy drugs, i.e. clofazimine, rifampicin and streptomycin, have no common features and therefore among themselves are compatible alternative drugs. Likewise, these three drugs are entirely different in their modes of action against bacteria in general, or Myco. leprae in particular, with the sulpha- or thioureagroups of antileprosy drugs. There have been many references to the use of combined therapy in leprosy in our discussions.

I have a feeling that many clinicians think of combined therapy primarily as a method for obtaining a significant increase in therapeutic activity and therefore as a means of obtaining more rapid cures. There is no significant evidence for this where combined therapy has been used in the chemotherapy of other bacterial infections, or in particular, where combined therapy is routinely used in tuberculosis. The paramount importance of using combined therapy, with striking advantage, is in reducing the incidence of drug resistance resulting from monotherapy, to insignificant proportions. The efficacy of combined therapy is based on sound bacteriological principles and is highly relevant to the chemotherapy of leprosy. Drug resistance results from the presence of a very small proportion of organisms in a bacterial population that are resistant to a particular drug and with the passage of time multiply sufficiently to repopulate the patient 
entirely with resistant organisms. The small proportion of such drug resistant mutants seldom exceeds one in a million $\left(10^{-6}\right)$. Therefore, if two drugs with entirely different modes of action are given at the same time, then at most the chances of resistant mutants occurring to both drugs would be at best not more than the product of two proportions, i.e. one in a million million $\left(10^{-12}\right)$. It is therefore on the basis of such an astronomically small proportion of dual resistant mutants existing in a bacterial population that combined therapy has proved highly beneficial. Clearly the advantage of combined therapy in almost completely excluding the possibility of the emergence of drug resistance, outweighs any small advantages which might result from the efficacy of combined therapy per se. These basic principles, which are highly relevant to the chemotherapy of tuberculosis, are likely to apply equally to the chemotherapy of lepromatous leprosy now that we know monotherapy results in drug resistance. Likewise, these basic bacteriological principles also explain why the need for using combined therapy applies only to patients with lepromatous type leprosy. While in the latter type of leprosy the bacterial population is high, and would be expected to contain a significant proportion of drug resistant mutants, in non-lepromatous leprosy the bacterial population is very much smaller and therefore few, if any, drug resistant mutants would be present.

My last point, though not directly concerned with drug resistance, is concerned with a basic principle pertinent to all trials. I refer here to the necessity of control trials for a meaningful assessment of any new drug or new combination of drugs. We have all heard in this Colloquium beneficial results being claimed for a new triple therapy, including rifampicin, from six Centres around the world. All six claim essentially rapid improvement, yet in none of these trials is this claim supported by a controlled comparison. Such an omission is unjustifiable in any circumstances, but is especially so in the particular trials undertaken because of the wide variations in type of leprosy and prior treatments, of the patients under study and because the triple therapies have included rifampicin. All six trials therefore have ignored the well-established knowledge on the variation in the response of patients within the leprosy spectrum, the problems associated with including treated and untreated patients in the same trial, and the well-established evidence based on control trials, which has already shown rifampicin, administered alone, to be more bactericidal than dapsone or any other antileprosy drug. Therefore, on the basis of these accepted principles, none of the claims being made for this triple therapy can be justified, or even accepted as being true, without including a properly matched group of patients treated by rifampicin alone. Regarding the problem of drug resistance in leprosy, this particular triple therapy regimen might well be advantageous, but from what we already know about resistance in leprosy, the type of controlled trials required would have to be undertaken on previously untreated patients and would have to continue for many years. The six trials on triple therapy fit none of these essential requirements.

\section{Prof. Freerksen}

In general, the most important reason for the use of combined therapy are the following:

(1) An intensification of the action reducing the time of treatment and thus avoiding relapses. 
(2) Up to now there do not exist any antimycobacterial medicaments inducing a bactericidal effect, but there are potential bactericidal substances. Bactericidal action can be approached by combining appropriate substances. It can easily be shown that rifampicin administered alone does not nearly attain the results achieved by rifampicin in combination with special substances (i.e. not all).

(3) Combined therapy ensures more safety regarding the therapeutic effect, because it prevents the development of resistant organisms and bacterial populations showing reduced sensitivity.

\section{Dr Browne}

Recalling WHO trials in which I was engaged 10 and more years ago, we were not able to prove any of those objects and the trials did not continue long enough to demonstrate the indefinite postponement of the emergence of resistant strains. We must still do a lot more work on this matter.

\section{Dr Gatti}

We have already over 100 patients with all types of leprosy, most of them being lepromatous cases, being treated with the combination of rifampicin plus dapsone. In 40 cases we gave a combination of dapsone plus clofazimine. We believe that combined treatment is useful, because it produces both clinical and bacteriological improvement and a lower incidence of leprosy reactions. When we used rifampicin, $600 \mathrm{mg}$ alone, reactions were very frequent. Up to now we have had no reactional episodes with this combination. In our experience with combined therapy, reaction is less intensive and we see also less risk of developing resistance.

\section{Dr Krenzien}

I should like to reply to Dr Rees' comments on our Borstel-papers this morning. I can only certify my own paper. It was not my intention to compare rifampicin and the combination therapy, which includes rifampicin and three other drugs. The major reason is the second one which Dr Pearson pointed out, and not to achieve a quicker elimination of the bacillary load of the patients under combined therapy. When we compare our results with those you obtained with rifampicin monotherapy, we may come to the conclusion that there is no difference in the speed of elimination of the bacillary load between single therapy and combined therapy. The major advantage would be the prevention of resistance. You did not have the same numbers as I had, but we use the same method, we counted the bacilli. You came to the result that you eliminate round about $90 \%$ in the first year and this is exactly what I found also.

\section{Prof. Azulay}

I think we have here a very important problem, but it is very difficult to say whether a drug combination is good or not. Each antibiotic has a special way of acting. Two bactericidal antibiotics help each other, sometimes a bactericidal plus a bacteriostatic drug help each other. It should be easy by laboratory trials to find out which combination is the most effective. But in leprosy we encounter a special problem because we do not know exactly in which phase of the bacterial life cycle the antibiotic is acting. This is why we can only guess or find out empirically by trials over a long period, which combination of drugs is good or 
not. Another point should be mentioned in this context, because it is of more interest than combined therapy, namely alternative treatment, sulphone after clofazimine, after rifampicin and so on. This problem has been neglected till now.

\section{Dr Gatti}

We had a few cases treated with clofazimine over a period of 24 months, in whom the Bacterial Index became negative. One patient under clofazimine treatment however still showed positive findings on bacilloscopy. Under combined therapy with dapsone and clofazimine over a period of six months, the findings on bacilloscopy became negative. I think that in this sphere many a question arises. One combination of drugs is not equivalent to another.

\section{Dr Browne}

Are you suggesting that this is a possible case of resistance to clofazimine?

\section{Dr Gatti}

I am not sure, but my experience is that this patient was a problem.

\section{Dr Browne}

Is there anybody else with similar experience of bacterial recrudescence during clofazimine therapy?

\section{Dr Molesworth}

We had a patient who was one of our control group on a $100 \mathrm{mg}$ of dapsone daily and who produced clinical resistance after two years, with the reappearance of solid staining bacilli, the reappearance of nodules and general degeneration. We gave her Lamprene (clofazimine), and clinically we immediately began to get a response, but no fall whatever occurred in the BI which was 5, and the MI remained steady at about 3 to $4 \%$. After another period of about two years, quite by chance, I suggested putting her on dapsone as well, and the combination produced an immediate result which is being maintained, so that both BI and MI have now fallen. Clinically she has maintained a very satisfactory response.

\section{Prof. Saerens}

I would like to raise another question in connection with combined therapy. If we do accept as logical the idea of combined therapy, but if we think on the other hand of the socio-economic aspects, the use of rifampicin would probably mean intermittent therapy. Not all drugs seem to be suitable as intermittent companion drugs. This problem should be investigated. We may expect this to be the case for the sulphones, but we really do not know. For tuberculosis it has meant a lot of work, and Prof. Mitchison in England has done a lot of work to find out suitable companion drugs in intermittent therapy. In this respect we hardly know anything in relation to leprosy. Intermittent therapy is likely to be one of the partial solutions to the problem of therapy in leprosy.

\section{Dr Urbancik}

I had the honour to serve in a WHO tuberculosis centre in South America and thus would like to stress the point that combined therapy might be of definite advantage in leprosy, because leprosy is very often found together with tuberculosis, and laboratory facilities in that part of the world and in developing 
countries are not good. It is often impossible to recognize tuberculosis but it is much more simple to recognize leprosy. Thus by combined treatment which is directed not against one but against more than one mycobacteriosis, at the same time, we can reach a better result.

\section{Prof. Freerksen}

In order to avoid any misunderstanding we need distinct definitions when discussing a problem. This especially applies to our definition of the terms "effect" or "cure". And we must also be well aware of the aim of our therapy. In my opinion we should distinguish at least three levels:

(1) Control of epidemics as leprosy control in the classical sense

(2) Individual treatment

(3) Eradication

These three objectives require different and not necessarily comparable procedures. Yet it is certain that the application of well-elaborated therapeutic methods at hospital level will be the best way to perceive what should be done in order to cure the individual patient and to prevent reinfections. An effective short-term therapy healing and simultaneously neutralizing the individual patient is the best protection against reinfection. The best epidemiological work can thus be done by means of highly effective therapy.

\section{II.4. Transfer Factor}

\section{Dr Browne}

Dr Ridley, will you please make some remarks on transfer factor?

\section{Dr Ridley}

I just thought as you mentioned this subject that I would mention a small study I have done recently relating the number of lymphocytes in skin lesions to immunological performance of the patient. A certain number of lymphocytes, not unexpectedly, are necessary to achieve any sort of good immunological performance. But unexpectedly over and above that level there is no effect, there is no relationship between the number of lymphocytes and performance, that is antibacterial performance. On the other hand a large number of lymphocytes does appear to give some sort of stability to a patient and prevent his downgrading in untreated patients or to increase the chances of upgrading with treatment. The point I want to make is that the number of lymphocytes is not an absolute number, but is related to the size of the lesion, and it seems to me therefore, that if one took a patient in the still fairly advanced stage of the disease, the dose of transfer factor required would be enormous and would have to be sustained. If there is a place for immuno-therapy in leprosy, it seems to me more likely that it would be found at a later stage of treatment when the disease has undergone regression and at that time it is possible that an effective upgrading or reversal reaction would be induced which might conceivably prevent a relapse.

\section{Dr Karat}

I have just two observations to make. In reference to Bullock's work in the United States, acceleration of the reversal process appeared after administration of 
transfer factor. I should like to ask Dr Ridley, whether he had the opportunity to distinguish between $\mathrm{B}$ and $\mathrm{T}$ lymphocytes in the context in which he described just now.

\section{Dr Ridley}

Just to answer Dr Karat's question. It is not possible to distinguish between $\mathrm{T}$ and B lymphocytes.

\section{Dr Rees}

From all the studies that I am aware of on the use of transfer factor in lepromatous leprosy, with one exception, all have shown minimal benefit. The one exception is the trial being carried out by Dr Hastings and his colleagues at Carville. In their studies they have, unlike the other trials, been administering transfer factor regularly over a significantly longer period, for many months. Their admittedly limited experience has shown that prolonged therapy with transfer factor resulted in a significant drop in the BI associated with a significant lymphocytic infiltration of the skin lesions.

\section{Dr Languillon}

It is very interesting to combine with chemotherapy a therapy which gives a stimulation of immunity. I have used a preparation named Ducton. I gave this by intramuscular injection, $5 \mathrm{ml}$ every two days to two borderline cases. When treating these two patients with dapsone, with sulphonamides, with Lamprene, I observed every time a borderline reaction with infiltration of lesions, ulceration and many bacilli in the nose and skin. I gave this treatment with Ducton alone during a period of two months. I obtained a total regression of the lesions and both nose and skin became bacteriologically negative in both cases. A friend of mine in Bamako used the same treatment in association with dapsone and obtained better results with the association of Ducton and dapsone than with dapsone alone. I think that this drug gives a very good stimulation of the property of macrophages in the treatment of leprosy.

\section{Prof. Azulay}

I agree with Dr Ridley that it is impossible to make a differentiation between $\mathrm{T}$ and B lymphocytes on the slides. But, those who have experience in the histopathology of leprosy know that there are some cases of lepromatous leprosy that have a huge number of plasmocytes; there are also other lepromatous cases that have few plasmocytes. As far as we know from immunological study, the plasmocyte is nothing else than a B lymphocyte that has changed its morphology. On the other hand, tuberculoid cases have no plasmocytes. Nevertheless we see one or two plasmocytes in slides from tuberculoid cases. I wonder if those cases with relapse have more plasmocytes than those who respond better to the treatment.

\section{Dr Browne}

I must say that I was certainly impressed by the histological evidence produced by our Korean colleague and also by the hint recently that there may be some drugs that will influence the development of $\mathrm{T}$ lymphocytes inducing them to take on unexpected properties which may be more that transient, but this work is still in the press. 